Int. J. Dev. Biol. 50: 523-532 (2006)

doi: $10.1387 /$ ijdb.052113ra

Original Article

\title{
Analysis of a homologue of the adducin head gene which is a potential target for the Dictyostelium STAT protein Dd-STATa
}

\author{
RYOTA AOSHIMA\#, RIEKO HIRAOKA\#, NAO SHIMADA\# and TAKEFUMI KAWATA* \\ Department of Biology, Faculty of Science, Toho University, Funabashi, Chiba, Japan
}

\begin{abstract}
A Dd-STATa-null mutant, which is defective in expression of a Dictyostelium homologue of the metazoan STAT (signal transducers and activators of transcription) proteins, fails to culminate and this phenotype correlates with the loss of expression of various prestalk (pst) genes. An EST clone, SSK395, encodes a close homologue of the adducin amino-terminal head domain and harbors a putative actin-binding domain. We fused promoter fragments of the cognate gene, ahhA (adducin head homologue A), to a lacZ reporter and determined their expression pattern. The proximal promoter region is necessary for the expression of ahhA at an early (pre-aggregative) stage of development and this expression is Dd-STATa independent. The distal promoter region is necessary for expression at later stages of development in pstA cells, of the slug and in upper cup and pstAB cells during culmination. The distal region is partly Dd-STATadependent. The ahhA-null mutant develops almost normally until culmination, but it forms slanting culminants that tend to collapse on to the substratum. The mutant also occasionally forms'fruiting bodies with swollen papillae and with constrictions in the prestalk region. The AhhA protein localizes to the stalk tube entrance and also to the upper cup cells and in cells at or near to the constricted region where an F-actin ring is localized. These findings suggest that DdSTATa regulates culmination and may be necessary for straight downward elongation of the stalk, via the putative actin-binding protein AhhA.
\end{abstract}

KEY WORDS: transcription factor, adducin, actin, cell differentiation, Dictyostelium

\section{Introduction}

Organized movement of various types of cells is necessary for the development of multicellular organisms (Hou et al., 2002; Baum, 2004). Such cell movements involve modulations of the cytoskeleton, such as the formation of actin stress fibers (Xia and Karin, 2004; Leptin, 2005). In order to understand the mechanism underlying such organized cell movement, the cellular slime mould Dictyosteliumserves as an excellent model. Dictyostelium cells aggregate by a chemotactic movement in response to cAMP to form a multicellular structure and the structure requires morphogenetic cell movement to complete development (Kimmel and Firtel, 2004).

The transcription factor Dd-STATa, a functional homologue of metazoan STAT (Signal Transducers and Activators of Transcription) proteins (Kawata et al., 1997), is necessary for efficient chemotactic cell movement at the aggregation stage and morphogenesis after the slug stage (Mohanty et al., 1999); suggesting that Dd-STATa may play a role in aggregation and multicellular development by regulating cell movement. The anterior prestalk region of the slug is composed of at least three subtypes of prestalk (pst) cells, pstA, pstO and pstAB cells. This is based on promoter analyses of two genes encoding extracellular matrix proteins, ecmA and ecmB (Jermyn et al., 1989; Gaskell et al., 1992). PstAB cells express both the ecmA and ecmBgenes (Gaskell et al., 1992) and are localized in a funnelshaped core of cells. The pstA cells express ecmA but not ecmB and $e c m B$ expression is actively repressed in the pstA cells (Ceccarelli et al., 1991; Harwood et al., 1993). The repression of $e \mathrm{~cm} B$ expression requires Dd-STATa; disruption of the $D d$ STATa gene causes the pstA and pstO cells to express ecmB (Mohanty et al., 1999). There is also a failure to culminate in the

Abbreviations used in this paper: ahh, adducin head homologue; Dd, Dictyostelium discoideum; EST, expressed sequence tag; pst, prestalk; STAT, signal transducers and activators of transcription.

\footnotetext{
*Address correspondence to: Dr. Takefumi Kawata. Department of Biology, Faculty of Science, Toho University, 2-2-1 Miyama, Funabashi, Chiba 274-8510, Japan. Fax: +81-47-472-5156. e-mail: tkawata@bio.sci.toho-u.ac.jp
}

\# Note: These authors contributed equally to this work. 
Dd-STATa null strain.

To further understand the molecular mechanism whereby culmination is regulated by Dd-STATa, we previously searched for Dd-STATa target genes that are expressed in the prestalk cells. These were analysed by microarrays and in situ hybridization (Maeda et al., 2003) and we identified 13 candidate genes (Shimada et al., 2004a). We previously analysed one of those genes, the SSK395(ahhA)gene. Here we show that $a h h A$ encodes a protein similar to adducin, an actin-binding protein. We identify promoter regions that regulate its expression, investigate protein localization in the culminant and discuss its function.

\section{Results}

\section{Prestalk cell-specific expression of the SSK395 gene}

In a previous study, we identified 13 prestalk-specific genes whose expression is down regulated in the Dd-STATa-null mutant (Shimada et al., 2004a). One of those is an EST clone SSK395, which is detectable at the tipped finger stage by in situ hybridization (Shimada et al.,2004a). We focused on the function of the gene encoding SSK395, because the expression pattern dramatically changes as development proceeds. At the

A

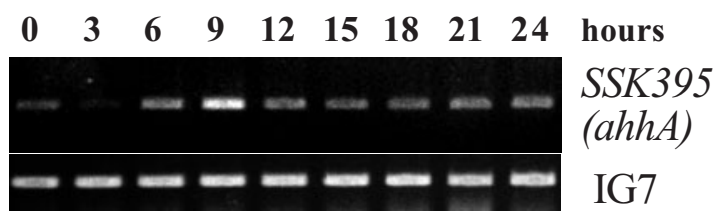

$\mathrm{Ax} 2$

\section{B}

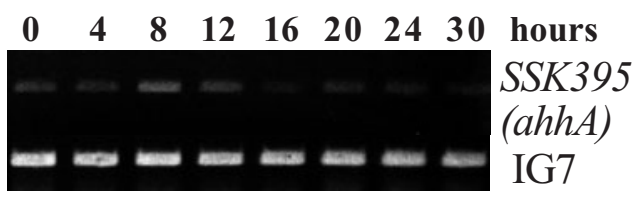

\section{Dd-STATa -}

Fig. 1. Comparison of ahhA(SSK395) gene expression in Ax2 and DdSTATa-null cells. (A) Developmental time course of ahhA(SSK395) gene expression in Ax2 detected by semi-quantitative RT-PCR. Total RNAs were extracted from $A \times 2$ cells at stages of growth phase (Oh), aggregates (3h, 6h), mound (9h), tipped aggregate (12h), slug (15h), Mexican hat (18h), culminant (21h) and fruiting body (24h) were used to amplify the specific ahhA(SSK395) DNA fragment as described in the Materials and Methods. (B) Developmental time course of ahhA(SSK395) gene expression in Dd-STATa-null mutant detected by semi-quantitative RT-PCR. Cells were at stages of growth phase (Oh), aggregates (4h, 8h), mound (12h), tipped aggregate (16h) and slugs (20, 24, 30h). IG7 was detected in the same reaction as a normalisation control. DNA was detectable after 19 cycles of amplification from $0.25 \mu \mathrm{g}$ total RNA before $P C R$ reaction saturated. Experiments $(A)$ and $(B)$ were performed at the same time with the same batch of reverse transcriptase to compare the amount of transcript. The experiments were repeated in triplicate and the representative data are shown. first finger stage, expression in prespore cells became almost undetectable although weak expression in prestalk cells is still seen. The expression level becomes elevated again at the slug stage and it is observed throughout the entire prestalk region. Once culmination begins, expression in pstA cells disappears and localisation of the transcript then gradually shifts to the upper cup cells and the stalk tube entrance in mid culminants (Shimada et al., 2004a).

Semi-quantitative RT-PCR was used to monitor the expression time course of the SSK395gene in Ax2 cells (Fig. 1A). In accordance with previous microarray observations (Iranfar et al., 2003), there are peaks of expression at 0 hour and 6 9 hours of development. SSK395gene expression is maintained at later stages of development (Fig. 1A).

\section{Reduced SSK395 gene expression in Dd-STATa-null mu-} tant at the late slug stage but not at the early stages

Interestingly, although expression at the tipped finger stage is detectable in the Dd-STATa null mutant, the transcript is hardly detectable in the mutant at the late slug stage (Shimada et al., 2004a). Therefore, only later expression appears to be positively regulated by Dd-STATa. To confirm whether expression of the SSK395 gene is Dd-STATa-dependent, we performed semi-quantitative RT-PCR in a Dd-STATa-null strain and compared expression to that in the parental strain, Ax2. SSK395transcripts were detectable in both strains at the tipped finger stage (9 hours of development for Ax2 and 12 hours for the Dd-STATa-null strain) (Fig. 1B). Interestingly, the transcript level at the mound stage in the mutant ( 8 hours of development) was rather stronger than that at the tipped finger stage. The expression level dropped during late first finger and early slug stages in both strains (12 15 hours of development for $A \times 2$ and 16 hours for Dd-STATa-null strain). Then, the expression increased slightly as development proceeded to the migratory slug stage in the Dd-STATa-null strain (20 hours of development).

\section{The SSK395 gene encodes a protein related to adducin}

The SSK395 gene contains an open reading frame (ORF), which is capable of encoding a protein of 268 amino acids with a predicted molecular mass of $30.1 \mathrm{kDa}$. A database search using BLAST showed that the SSK395gene encodes a protein with high homology to mammalian adducin (Fig. 2A). However, the gene does not encode the whole of adducin, it only encodes a domain corresponding to the head domain (Fig. 2B). Therefore, we named the SSK395 gene ahhA (adducin head-homologue $\underline{A}$ ).

\section{Promoter fragment of the ahhA gene confers prestalk- specific expression}

Comparison of $a h h A$ gene expression between $A \times 2$ and $D d$ STATa - null strains by in situ hybridisation (Shimada et al., 2004a) and semi-quantitative RT-PCR (Fig. 1) imply the presence of at least two regulatory promoter elements; one is for the early stages and is independent of Dd-STATa and the other is for the later stages and is partly Dd-STATa-dependent. To clarify whether this is true for ahhA gene expression, we amplified a promoter fragment of the $a h h A$ gene and subcloned it into pDd-gal17( $\mathrm{H}+)$ to make a lacZ-fusion reporter construct 


\section{A}

AhhA

$\alpha$-adducin

1 MNGDSRAAVVTSPPPTTAPHKERYFDRVDENNPEYLRERNMAPDLRQDFNMMEQKKRVSM 60

AhhA

$\alpha$-adducin

1

61 ILQSPAFCEELESMIQEQFKKGKNPTGLLALQQIADFMTTNVPNVYPAAPQGGMAALNMS 120

$:^{\star}$

AhhA

$\alpha$-adducin

4 NNKFTSSDDLSLVNKTKYSDEEYLVRVKLAATYRMVAHLGWDELIYNHLTARVPG-TDHM 62 121 LGMVTPVNDLRGSDSIAYDKGEKLLRCKLAAFYRLADLFGWSQLIYNHITTRVNSEQEHF 180

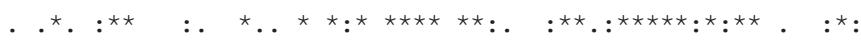

AhhA

63 LLNAFGMRFDEVTATSLVTIDMDGKIIDAGSTDLGINKTGYVIHGAIHKARPDILATMHV 122

$\alpha$-adducin 181 LIVP

\section{AhhA}

123 HQEDTVAVACYKEGLLPISQNSFIIGDISYHNYEGISINAQEQERIVKSLGPVNKNLILR 182

$\alpha$-adducin 241

$*$

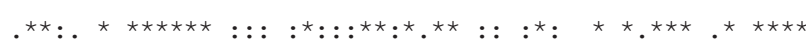

AhhA 183 NHGIVSCGNSIEEAFFFLYQLVKTCEIQVKMLSMVGGDISKLDIPDKQIRDFSTRTAASF 242 $\alpha$-adducin 301 NHGLVSVGESVEEAFYYIHNLVVACEIQVRTLASAGGPDNLVLLNPEKYKAKSRSPGSPV 360

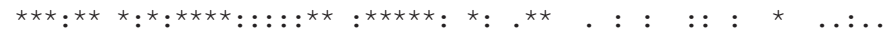

AhhA 243 TKQGNGKKEWKSYYRIVEKLDDSEKN 268

$\alpha$-adducin 361 GEGTGSPPKWQIGEQEFEALMRMLDNLGYRTGYPYRYPALREKSKKYSDVEVPASVTGYS 420 : $\quad$ : $*: \quad: . * *: . *$

\section{B}

Dd AhhA

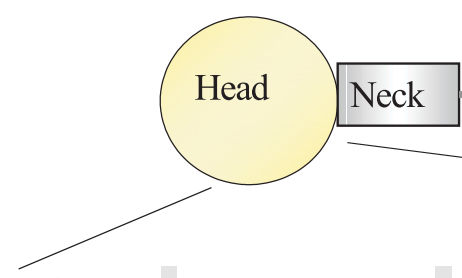

$\alpha$-Adducin 138 YDKGEKLLRCKLAAFYRLADLF

$\beta$-Adducin 126 LAKGERLMRCKISSVYRLLDLY

$\alpha$-Actinin 77 LAKPER-GKMRVHKISNVNKAL

$\beta$-Spectrin 99 LPKPTK-GKMRIHCLENVDKAL

Dystrophin 62 L--PEK-GSTRVHALNNVNKAL

Hs ABP280 91 RKHNQR-PTFRQMQLENVSVAL

Fimbrin

Consensus

$$
\text { ..k.e...........al }
$$

182 INKKKL-TPFTISENLNLNLAL
Fig. 2. Homology of the ahhA gene product with $\alpha$-adducin, together with a scheme of the adducin protein and alignment with a putative actin-binding domain. (A) Alignment of the AhhA amino acid sequence with human $\alpha$ adducin 1 laccession number L29296. 1; Lin et al., 1995). Amino acid sequences of AhhA and human $\alpha$-adducin 1 were aligned by using ClustalW program. Asterisks indicate identical amino acid residues between human $\alpha$-adducin 1 and AhhA. Similarly, colons denote the amino acid residues with conservative change; dots indicate the residues with semi-conservative change. Amino acid position numbers are shown at both ends. Only the N-terminal portion corresponding to 420 residues is shown for human $\alpha$ adducin 1. (B) Schematic representation of adducin protein and an alignment of a putative actin-binding domain. The schematic structure of the $\alpha$-or $\beta$-adducin subunit is shown at the top ladapted from Hughes and Bennett, 1995; Li et al., 1998). Adducin consists of head, neck and Cterminal tail regions. AhhA spans a region corresponding to head region of adducin. A sequence alignment of a putative actin-binding domain with conserved 27 amino acid-domain of already known actin-binding proteins and AhhA is shown at the bottom. Sequences of human $\alpha$-adducin, human $\alpha$-actinin (accession number P12814), human $\beta$-adducin (accession number P35612), Dictyostelium AhhA (accession number C91531), human $\beta$-spectrin (accession number P11277), human Dystrophin (accession number P11532), human endothelial ABP-280 laccession number P21333) and chicken fimbrin (accession number P19179) were aligned according to MultAlin program (Corpet 1998; http://prodes.toulouse.inra.fr/ multalin/cgi-bin/multalin.pl). Conserved amino acids are shaded. Hyphens indicate gaps of sequence alignment. For symbols of consensus sequence, ! is either I or V, \$ is either L or M, \% is either $F$ or $Y$ and \# is either $N, D, Q, E, B$ or $Z$. Amino acid position numbers are shown at both

\section{ends.}


A

1120 TAGAAGATGATGTAGTAATACCTAATCTTAATAATTCACCATTAG - 1081

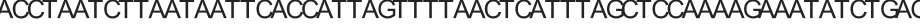

- 1040 ATTGAATCTATTATGTCTAATAAATAAAATTATTATTATTATTATTATTATTATTATTATTATTATTATTATTATTATTA

- 960 TTATTATTATTATTATTATTATTATTATTATTATTATTATTATTATTATTATTATTATTATATTTTATTTTATTTTTTAAAA

- 880 ATTAATAATAATAAAAAAAAAAAAAATCATATTTTTTAAAGT GTTTAGAAAAAAAAAATCATATTTTTTTAAAGTGTTTAG

- 800 AAAAAAAAAATTAAAAAATAAATAAAAAAAAATATATATTAGTAATTATCTCAAATAATAAGATTAATTTAGGTGTAAAA

- 720 AAAAAATAAGAAGAGATTGGGTTAGGTTGGGGGAGAGAGAGAATATTCAGATTTGTTTTTTTTTTCTTTTTTTTTTTTTCT

- 640 TTTTTTTTTTTTTTTTTTAAATCTTTATTATTATTATTATTATTATTATTATTATTATTATTATTATTATTATTATTATTA

- 560 TTATTATTATTATTATTATTATTATTATTATTATTTTTATTATTATTTTTTTAAAAAAAAAAAAAAAAAAACGATACATTC $\longrightarrow-473$

- 480 CTATAGTTTACCCCCTTTTGAGTATACTAAACTTTTTTTGATT GGTTTGGAAAAAAAAAAATAAATATAATATTAAATTTT

400 TTTTTTTTTTTCCTTTTAAAAAATATAGATATTATTGAATATTATTTTAATTTTTTGATTATTAAAAAACATAAAAAATG

- 320 ATGGGACAGGGAGTTAATTATTTTTTTTAATTTTAAAATACAAATTATTTTTTTATATTTTTTTTTATTTTTTTTTTATTTTT

240 - 174 $\rightarrow-132$

- 160 ATAAAACTACTTAAAAAAAAAAAAAAAAAATGAAAAAAATAACAAAACATTGTTTTTTTTTTTTTTTTTTAATTTAATATTC - 80 AATTTTATTTTTTTTTAAATCTTAATCATTATAAAATCAAAACAAATAAAATAAATAAAAAAAATAAAATTTAATATAAAA +1 ATG

B

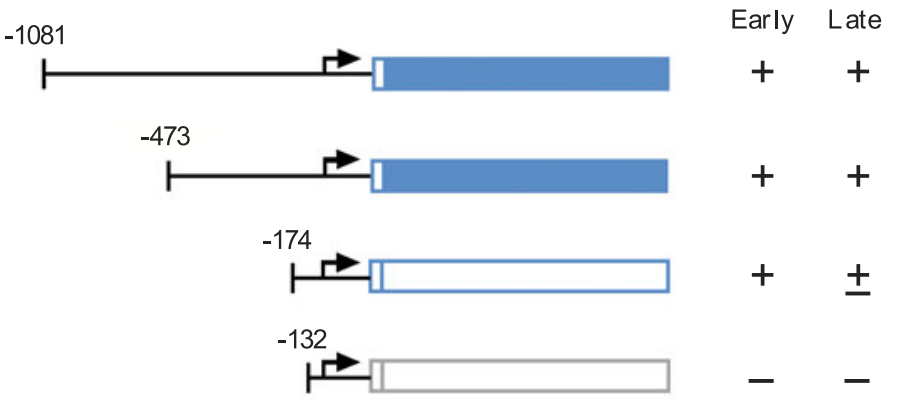

and the promoter activity was assayed by its $\beta$-galactosidase activity.

A DNA fragment, which has a 5' end-point 1081 bp upstream of the putative translational start codon of the $a h h A$ gene $(a h h A-$ 5 ' $\Delta-1081:: / a c Z$, see Fig. 3), gave a similar staining pattern to that observed in the in situhybridisation experiment (Shimada et al., 2004a). The signal was detected during aggregation (Fig. 4Aa). Later, a signal became detectable in the pstA region at the tipped mound and slug stages (Fig. 4A b,c,d). At the Mexican hat stage, cells expressing lacZ are found in the tip region (Fig. 4A e). The stained cells eventually locate as a thin ring-shaped structure in the prestalk region, stalk tube entrance and disk cells (Fig. 4A f,g).

Unlike the in situhybridisation results (Shimada et al., 2004a), the signal was also detected in the Dd-STATa-null mutant transformed with ahhA-5's-1081.:/acZ construct throughout entire development (Fig. 4B). This may reflect traces of $\beta$-galactosidase activity during the early stages of development because of its stable character. Therefore, we replaced the lac $Z$ gene with ile:: $/ a c Z$, a fusion gene that produces unstable $\beta$-galactosidase (Detterbeck et al., 1994) and compared the staining pattern between in Ax2 and Dd-STATa-null strains (Fig. 5).

The construct showed weakened staining, particularly in pstA cells, at late stages of development in the Dd-STATa-null strain
Fig. 3. Analyses of the $a h h A$ gene promoter. (A) Nucleotide sequence of the ahhA gene promoter region. Positions of nucleotide numbers are shown to the left. The position of a putative translational start site is referred to as +1 and is boxed. The positions of each deletion points at $-1081,-473,-174$ and -132 are indicated by arrows. The region with shaded letters is necessary for the prestalk-specific expression at multicellular stages of development (see Fig. 6). (B) Schematic representation and summarized expression of each $5^{\prime}$ deletion construct. Expression at both early stages (until tip formation) and late stages are shown schematically as + (expressed), \pm (barely detectable) and - (not expressed). compared to the staining of $\mathrm{Ax} 2$ strain (Fig. 5B), implying the involvement of Dd-STATa in the expression of the ahhA gene at late stages of development but not at early stages as already seen in the in situ and RT-PCR experiments (Shimada et al., 2004a, Fig. 1). There is still a discrepancy between RT-PCR data and unstable lac $Z$ data because of the absence of expression of the unstable $\beta$-galactosidase before tipped aggregates (Figs. 1 and $5)$. If a half-life of unstable $\beta$-galactosidase is the same, it could be possible that the stability of ahhA mRNA may change depend on developmental time course, although the precise reason is unknown.

\section{Mapping of the ahhA promoter regions necessary for early and late stage-specific expression}

To identify the regions necessary for expression at early and late stages, a series of 5'-3' deletion constructs were created. Deletion to $712 \mathrm{bp}$ upstream of the putative translational start codon (termed ATG hereafter) weakened the staining in all pst cells at the slug and culminant stages in Ax2 and at the slug stage in the Dd-STATa-null mutant (data not shown). Further deletion to $473 \mathrm{bp}$ upstream of the ATG caused ectopic expression of the lacZ gene in the late prespore/spore region of the culminats in addition to the prestalk staining of slugs (Fig. 6A). This indicates that a late prespore/spore-specific repressor region is located between nt (nucleotide) -712 and $\mathrm{nt}-474$.

Cells transformed with construct $a h h A-5^{\prime} \Delta-174:: / a c Z$ showed no pst- nor late staining. The observed diffuse staining (not a specific pst-staining) may due to scattered cells expressing stable $\beta$-galactosidase from beginning of development or pre-aggregation stage (Fig. 6B). Cells transformed with construct $a h h A-5$ ' $\Delta$ 132::/acZ lacked staining entirely at all stages of development (Fig. 6C). These results demonstrate that the region necessary for expression at the later stages in pstA cells is between nt -473 and -175 , while expression at the early stages requires sequences between $n t-174$ and -132 .

Disruption of the ahhA gene leads to a tendency to form slanting culminants and aberrant papillae

To determine the function of the AhhA protein, mutants lacking 
A

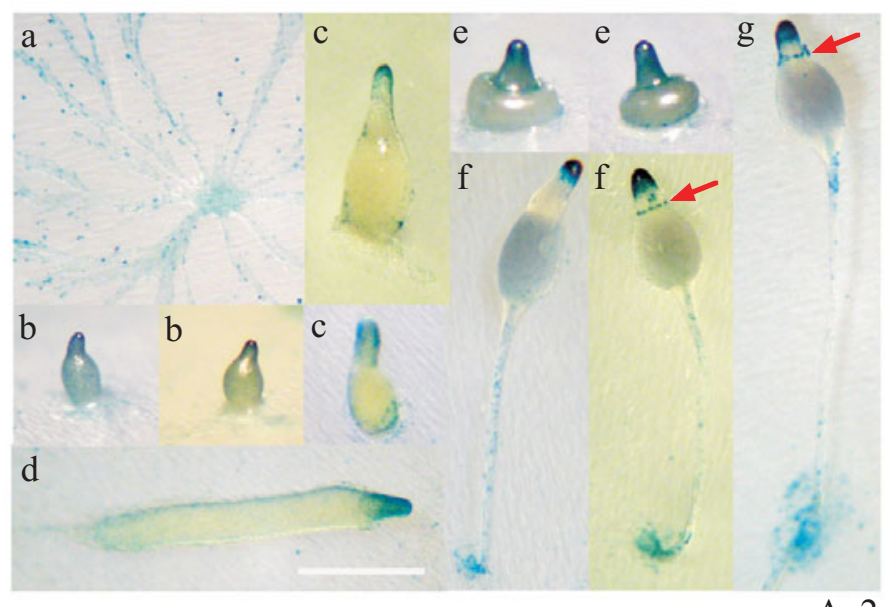

B

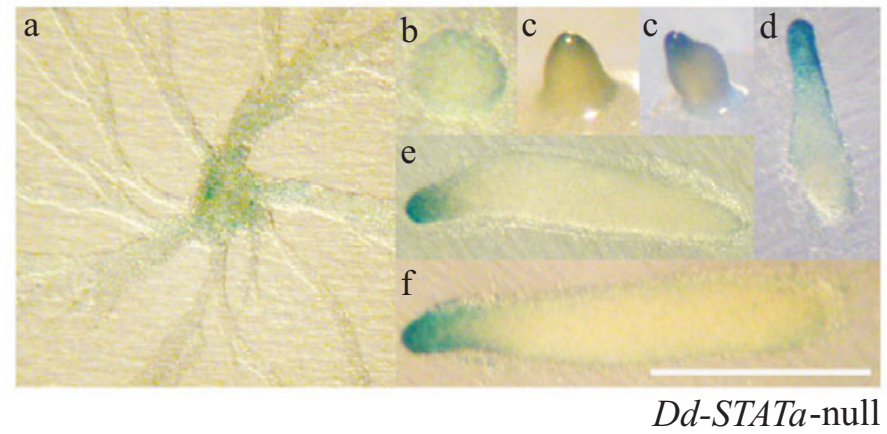

Fig. 4. Spatial expression pattern of ahhA promoter activity detected by lacZ reporter construct. (A) Ax2 cells transformed withahhA(WT)::lacZ, which contains a $1081 \mathrm{bp}$ upstream promoter region fused to $\beta$-galactosidase (lacZ) gene, were allowed to develop on filters to stages of aggregation stream (a), tipped finger (b, c), slug (d), Mexican hat (e), mid culminant (f) and late culminant (g). Bar indicates $500 \mu \mathrm{m}$. Arrows indicate the ring shaped staining specific to late stages of development (see text for details). Staining was performed at $37^{\circ} \mathrm{C}$ for 30 min to detect ahhA-expressing cells. (B) Dd-STATa-null cells transformed with ahhA(WT)::lacZ were allowed to develop on filters to stages of aggregation stream (a), mound (b), tipped finger (c) and slug (d-f). Bar indicates $500 \mu \mathrm{m}$. Staining was performed at $37^{\circ} \mathrm{C}$ for $\underline{45}$ min to detect ahhA-expressing cells.

the ahhA gene were created by homologous recombination (Fig. 7A). Two independent targeted clones were isolated by PCR screening using genomic DNA as a template and a semi-quantitative RT-PCR analysis showed that the ahhA transcript is almost undetectable (Fig. 7A). When the $a h h A^{-}$cells were allowed to develop on either phosphate-buffered filters or on water agar plates, the $a h h A$ cells developed more rapidly, by approximately one hour (data not shown), but morphologically normally until early culmination (data not shown). At the mid stage of culmination, $\sim 5 \%$ of the mutants formed swollen papillae with a constriction at the prespore-prestalk boundary (Fig. 7B c,d). The terminal structure of most mutant entities was indistinguishable from that

\section{A}

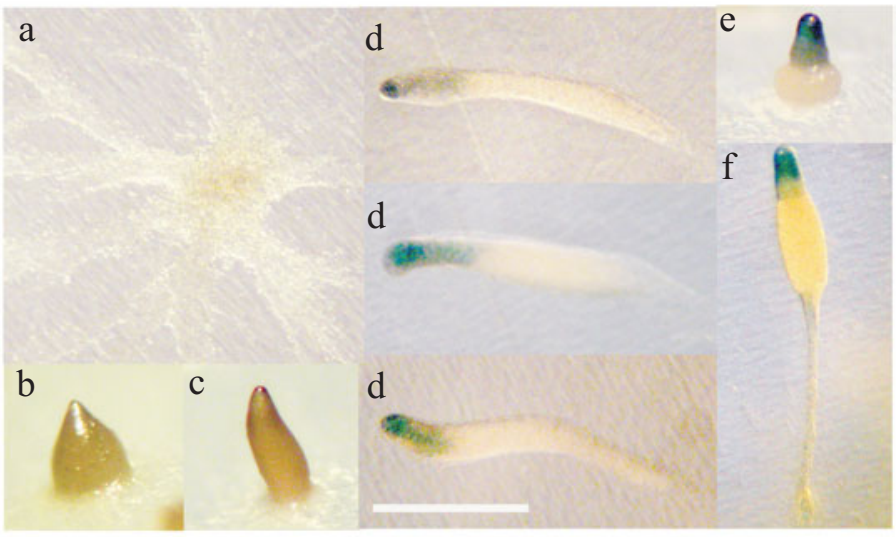

Ax2 of the parental strain, Ax2, except that the mutant formed a slanting fruiting body and easily collapsed on to the substratum (Fig. $7 \mathrm{Be}$ ). This latter, weaker phenotype is more prevalent, as more than $50 \%$ of the culminants show such behaviour (data not shown). All these phenotypic defects were rescued by expressing AhhA protein under the control of its own promoter (Fig. 7Bf). Therefore, all defects of the mutant are attributed to inactivation of the ahhA gene.

Since several null mutants of genes encoding actin-binding protein show a more severe phenotype when they are allowed to develop on soil (Ponte et al., 2000), we tested the phenotype $a h h A^{-}$cells on soil plates. However, no phenotypic change was observed in such experiments (data not shown).

\section{Localization of the AhhA protein at the tip, pstO cells and cells near the constricted region}

In order to elucidate AhhA function, we made a construct, which expresses AhhA protein with a human c-myc epitope tag, under the control of the $a h h A$ promoter (Fig. 8A). The construct was transformed into the ahhA-null mutant and AhhA protein

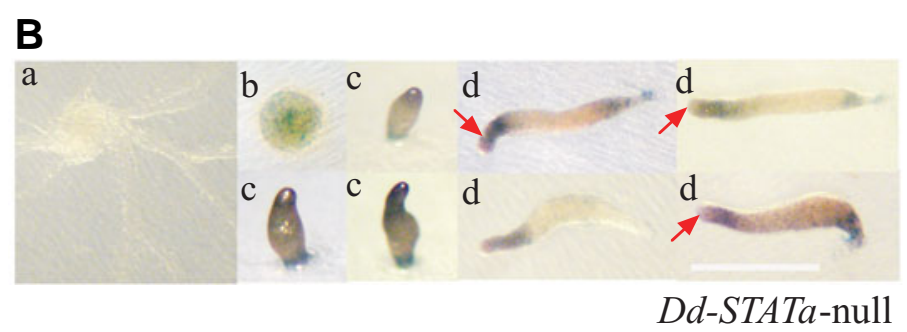

Fig. 5. Spatial expression pattern of ahhA promoter activity detected by ile::lacZ reporter construct. (A) Ax2 cells transformed with ahhA(WT):: ile ::lacZ, which contains an 1081 bp upstream promoter region fused to unstable $\beta$-galactosidase (ile ::lacZ) gene, were allowed to develop on filters. Stages are the same as described in the legend of Fig. $4 \mathrm{~A}$. Bar indicates $500 \mu \mathrm{m}$. Staining was performed at $37^{\circ} \mathrm{C}$ for $\underline{3}$ hours to detect ahhA-expressing cells. (B) Dd-STATa-null cells transformed with ahhA(WT)::ile ::lacZ were allowed to develop on filters. Stages are the same as described in the legend of Fig. 4B. Bar indicates $500 \mu \mathrm{m}$. Staining was performed at $37^{\circ} \mathrm{C}$ for $\underline{5}$ hours to detect ahhA-expressing cells. Arrows indicate the weakened staining of pstA cells in the Dd-STATa-null mutant (see text for details). After prolonged incubation, the staining before tipped aggregates becomes visible, thus to be expressed at a very low level. 
A

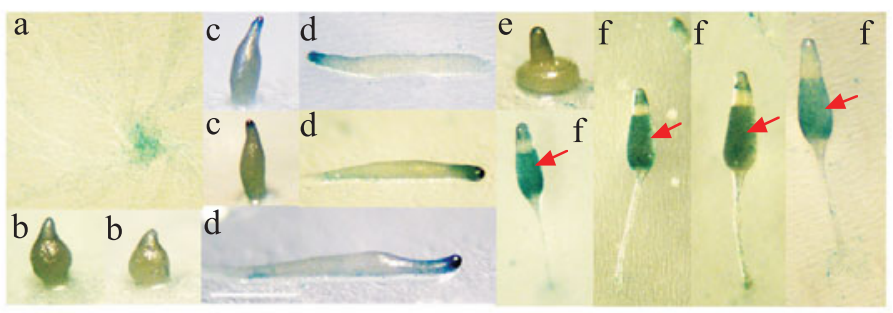

$\operatorname{ahhA}\left(5^{\prime} \Delta-473\right):: 1 a c Z$

B

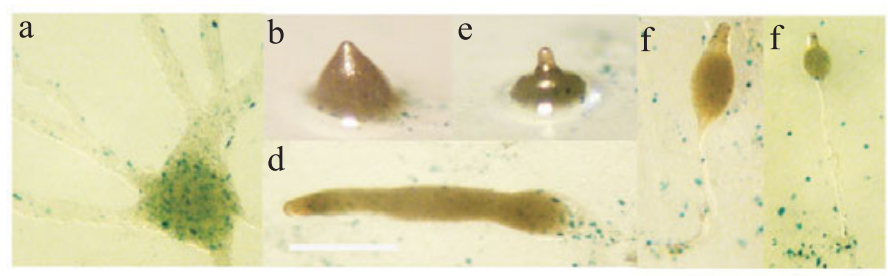

$\operatorname{ahhA}\left(5^{\prime} \Delta-174\right):: \operatorname{lac} Z$

C

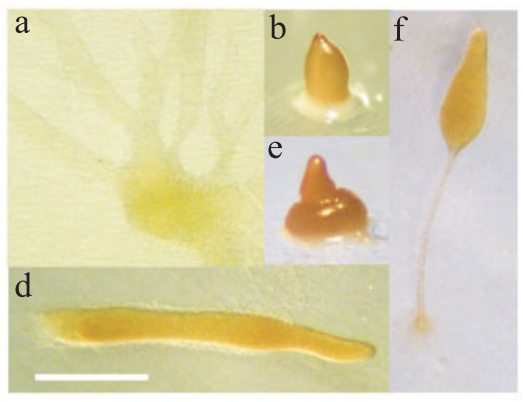

$\operatorname{ahhA}\left(5^{\prime} \Delta-132\right):: 1 a c Z$

Fig. 6. Deletion analysis of the ahhA promoter in Ax2 cells. Expression patterns are shown for the deletion constructs (A) $\Delta-473$ ::IacZ, (B) $\Delta-174::$ lacZ and (C) $\Delta-132:$ :lacZ. Transformed cells with each lacZ construct were allowed to develop on filters. Stages are the same as described in the legend of Fig. 4A. Bar indicates $500 \mu \mathrm{m}$. Staining was performed at $37^{\circ} \mathrm{C}$ for 1 hour for $(A)$ and overnight for $(B)$ and $(C)$ to detect ahhA-expressing cells. Arrows indicate the ectopic staining in the psp cells for the $\Delta-473:$ :lacZ construct.

expression was monitored. Western blotting of total cell proteins from three independent clones identified a protein with an approximate molecular weight of $30 \mathrm{kDa}$, which matched well to the size predicted from the AhhA amino acid sequence (Fig. 8B).

Next we examined AhhA protein localization by immunohistochemical staining (Fig. $8 \mathrm{C}$ ). The protein localized at the stalk tube entrance and pstO cells (arrowhead). The protein also localized in cells near the constricted region, where the $a h h A$ transcript localized (Shimada et al., 2004a; Fig. 4A). Since the AhhA has a putative actin-binding domain and the F-actin ring localizes at the constricted region, we tried co-staining with fluorescent phalloidin. As shown in Fig. 8C, AhhA protein colocalized with $\mathrm{F}$-actin at the cells near the constriction (coloured arrows).

\section{Discussion}

\section{A new Dd-STATa-dependent marker expressed in prestalk cells}

In this study we identify a new marker gene, $a h h A$, which is expressed in all cells at the early stages and comes to be expressed in prestalk cells at later developmental stages, after the tipped finger stage (Fig. 4A). Expression in pstA cells at stages later than the slug stage is down-regulated in the $D d$ STATa-null mutant, while expression up to the tipped finger stages is not (Shimada et al., 2004a; Figs. 1 and 5). The spatial and temporal patterns of $a h h A$ expression look almost the same in Ax2 and Dd-STATa-null cells but longer staining is required to visualize it (Figs. 1 and 4). However, lacZ staining in pstA cells is down-regulated in the Dd-STATa-null mutant compared to the staining in pstO cells (see Fig. $5 \mathrm{~d}$ ), The prestalk marker gene ecmA shows a similar expression pattern at the slug stage but there is a critical difference in the expression patterns of these two genes during culmination. During culmination, the ecmA gene is expressed in all prestalk cells. However, expression of the ahhAgene in the pstA cells disappears and then reappears in the upper cup cells, pstAB cells, stalk tube entrance and disk cells. There is no detectable expression in the bottom part of the stalk (Fig. 4A). This expression pattern is rather similar to the ecmBgene expression pattern. However, there is a difference in the expression pattern in the late culminant. Unlike the $a h h A$ gene, the $e c m B$ gene is expressed in the entire stalk. Most notably, the ecmB gene is also expressed in the lower cup cells. Therefore, the $a h h A$ gene is a novel type of prestalk marker gene whose expression is partly dependent on Dd-STATa. It provides a tool to investigate the function of DdSTATa.

\section{Subregions of the ahhA promoter directing early and late stage expression}

Although the ahhAgene is expressed throughout development, the transcript amount and spatial expression pattern of ahhA show very complicated profiles (Shimada et al., 2004a; Figs. 1A, 4A). Deletion analysis of the $a h h A$ promoter mapped a region necessary for expression in prestalk cells (particularly in pstA cells) at "later stages" to between -473 and -175 , a region necessary for expression at early stages to between -174 and -133 (Figs. 3 6). A region between -712 and -474 must have a negative regulatory element(s) repressing $a h h A$ expression in late prespore/ spore cells and a region between -473 and -175 may contain a region required for the expression in those cells (Fig. 6A). The expression pattern of the $a h h A-5$ ' $\Delta-473:: / a c Z$ deletion construct in those cells is similar to that observed for spiA gene, a marker gene of late prespore/spore cells (Richardson et al., 1994).

Since 5'-deletion constructs for further fine mapping are all unstable in bacterial cells, we have been unable to identify sequences necessary for prestalk cell expression at later stages in the region between -473 and -175 (data not shown). There are several GC sequences in this region (Fig. 3). Placing such GC sequences upstream of the $a h h A-5$ ' $\Delta-174: \because / a c Z$ deletion construct gave no staining (data not shown). Therefore, it is likely that at least two GC sequences in this region are necessary for prestalk specific expression and the sequence element 


\section{A}

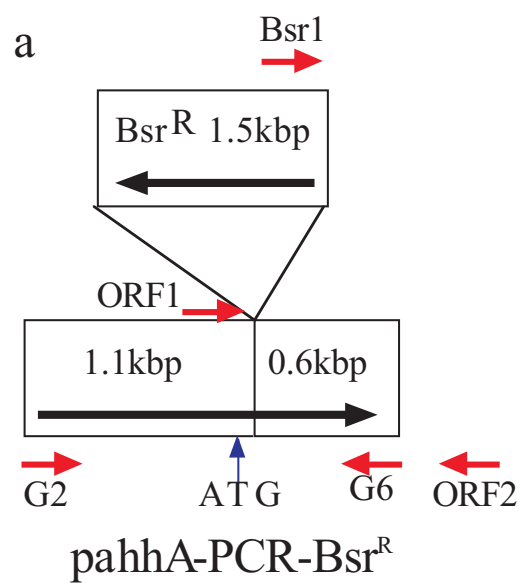

b

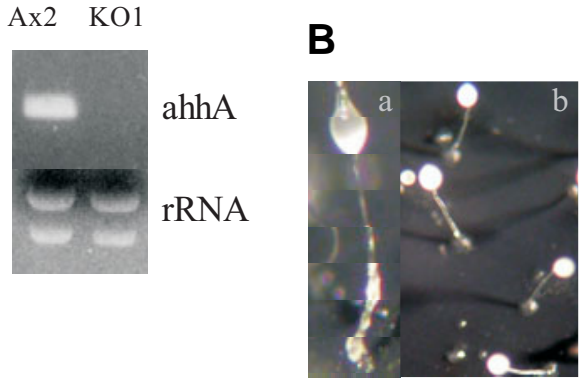

Ax2

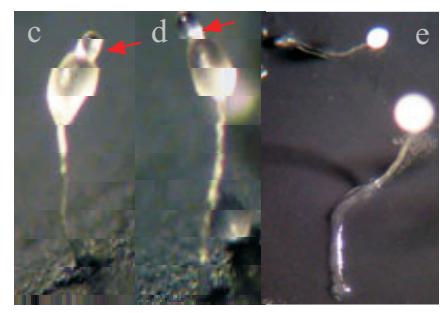

$\operatorname{SSK395(ahhA)}$ null mutant

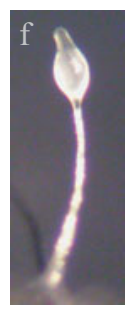

rescued

Fig. 7. Knock out mutants of ahhA gene. (A) Creation of ahhA knock out construct and screening of ahhA- strains. Knock out construct of ahhA gene. $B \mathrm{Br}^{R}$ cassette was inserted as described in Materials and Methods at 81 bp down stream from the ahhA gene translational start site (panel a). Targeted gene disruption was screened by genomic PCR with primer pair, Bsr-1 and 395-ORF-2 (data not shown). The disruption was further confirmed by both genomic PCR (data not shown) and RT-PCR (panel b). Genomic PCR was performed with primer pair 395-G2 and 395-G6 for two independently isolated ahhA-null mutants (KO1 and KO2). One microgram of total RNAs isolated from wild-type (Ax2) and the ahhA-null mutant (KO1) at vegetavely growing stage were used for RT-PCR. Ribosomal RNAs (1 $\mu \mathrm{g}$ total RNA/lane) were electrophoresed and stained with EtBr for loading standard. (B) Developmental phenotype of ahhA-null mutant. Cells of wild-type (Ax2, panels a and b), the ahhA-null mutant (panels c-e) and the rescued strain (panel f) were allowed to develop on phosphate-buffered filters to mid-culmination stage (panels $a, c, d$ and f). Panels $b$ and e show the overhead views of the late-culminants of the wild-type (Ax2) and the ahhA-null cells, respectively. Arrows indicate the swollen papillae of the mutant (see text for details).

A

B

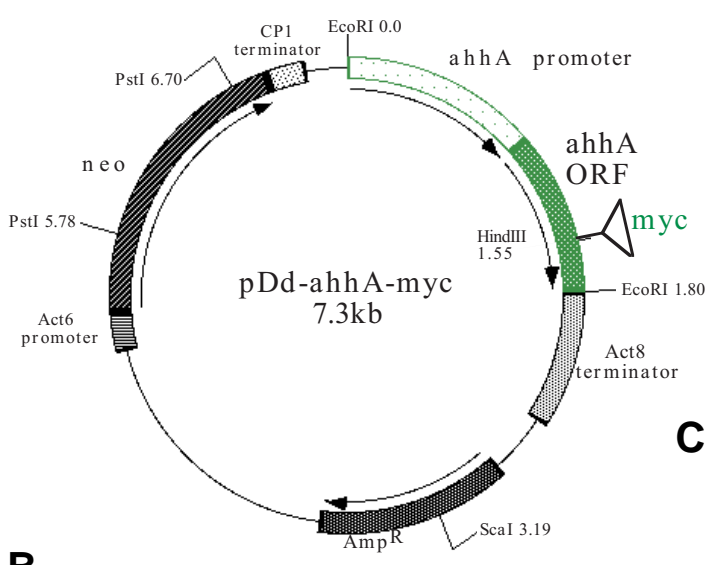

$\begin{array}{lllll}\mathrm{kD} & 1 & 2 & 3 & 4\end{array}$

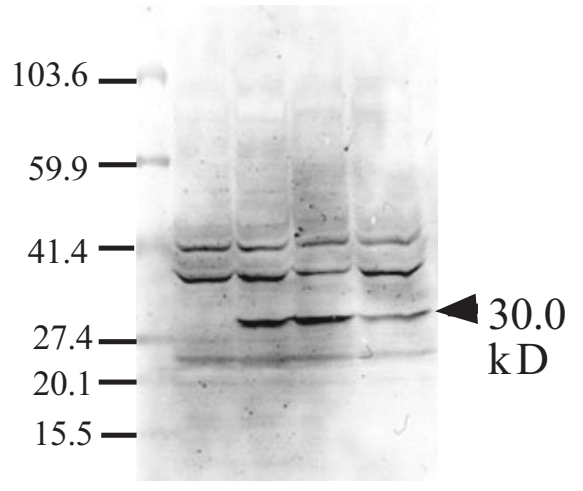

Fig. 8. Detection of the AhhA protein in the culminants. (A) Expression construct of the AhhA protein, $p D d$-ahhA-myc. The ORF region of the ahhA gene was placed under the control of its own promoter. Human c-myc epitope tag was introduced into the unique Hindlll site in the coding region. (B) Expression of the AhhA protein as detected by Western blotting. pDd-ahhA-myc was transformed into ahhA-null cells and three independent transformants $(r 1, r 2, r 3)$ were analysed. For Western analysis, approximately $20 \mu \mathrm{g}$ of total cell protein was loaded per each lane and analysed as described in Materials and Methods. Lane 1 indicates Ax2 proteins, lanes 2-4 correspond to the transformants 1 1-r3, respectively. A protein band with a molecular weight of approximately $30 \mathrm{kDa}$ (arrowhead) was visible in lanes of transformants but not of Ax2, thus, supposed to be specific AhhA band. (C) Spatial expression of the AhhA protein as detected by immunohistochemical staining. Photographs (a) and (c) are relief contrast images, $(b, d, e, f)$ are fluorescent images of the mid-culaminants of $A \times 2$ strain $(a, b)$ and
C

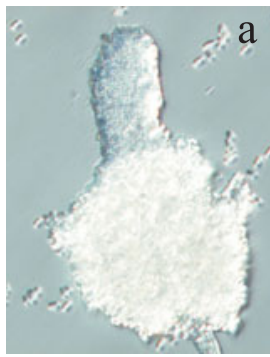

a

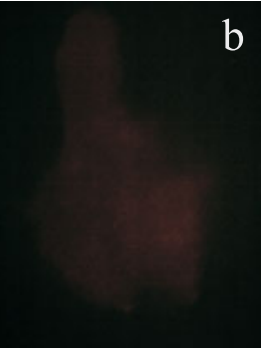
AhhA::c-myc expressing strain (c-f). (b,d) are images of anti-c-myc antibody detected by Alexa Fluor 568 labelled secondary antibody (Invitrogen). (e) is an image of F-actin detected by Alexa Fluor 488 phalloidin (Invitrogen). Coloured arrows indicate localization of AhhA protein near the constricted region of lateculminats; the arrowhead indicates pstO localization of the AhhA protein (see text for details).

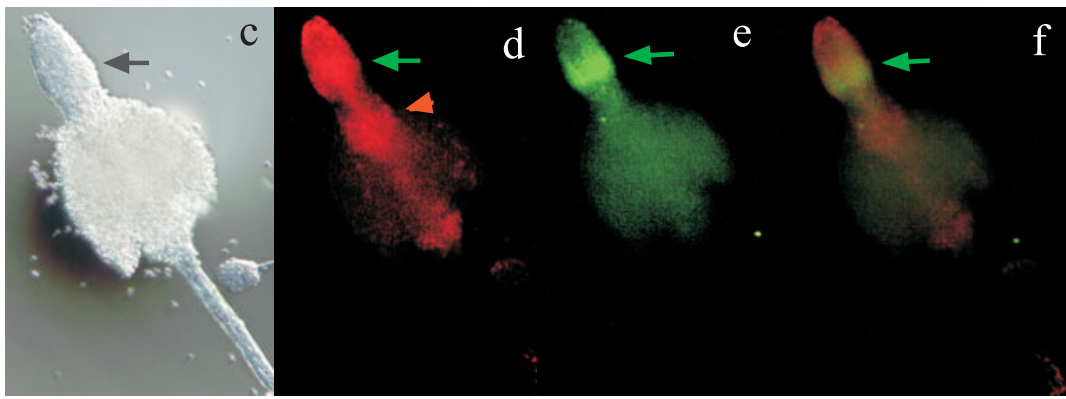


responsible for expression in prestalk cells remains to be identified.

\section{Putative function of the AhhA protein}

AhhA is a protein with a putative actin-binding domain and it is highly homologous to mammalian adducin (Fig. 2). Data base search shows that there is no other gene encoding an adducin-like protein in the Dictyostelium genome (Eichinger et al., 2005). Adducin is a heterodimeric cytoskeleton protein involved with actin and spectrin in forming specialized contact sites in various multicellular organisms (Yue and Spradling, 1992; Robinson et al., 1994). Interestingly, the AhhA protein lacks domains corresponding to neck and protease-sensitive carboxy-terminal tail but harbors a globular head domain (Hughes and Bennett, 1995). The C-terminal tail is a target of protein kinase $C$ and Rho kinase and has an important role in forming a complex with fast growing ends of actin filaments that recruit spectrin (Hughes and Bennet, 1995; Li et al., 1998). Therefore, the AhhA protein may not be an authentic adducin.

Despite lacking the C-terminal domain of adducin, localization of AhhA protein is in cells where F-actin is enriched and where there is a constriction in the stalk tube (Grimson et al., 2000; Fig. $8 \mathrm{C})$. Although there is no evidence to show a direct interaction of AhhA protein with F-actin, the above result and the presence of putative actin-binding domain imply that the AhhA protein may interact with F-actin.

Inactivation of the ahhA gene causes almost no phenotypic change but ahhA-null cells form aberrant culminants: slanting culminants, frequently than is observed for wild-type cells (Fig. 7C). The mutants easily collapse on to the substratum (Fig. 7Be). Under microscopic observation the mutant stalk is indistinguishable from that of wild-type stalk (data not shown), therefore, the collapse may be caused by the slanting of culminants but not by the weakness of the stalk. In addition, the AhhA protein co-localizes at a region near or where the stalk tube is constricted. This may indicate that AhhA is necessary for the correct downward elongation of the stalk. It may control the shape of the culminant through interaction with other molecules, possibly F-actin or adherens junction molecules (Grimson et al., 2000; Coates and Harwood, 2001; Coates, 2003; Williams and Harwood, 2003). A protein supposed to be adducin has been identified in Physarum polycephalum. It forms large aggregates of actin filaments and modifies actin-myosin interaction by blocking it (Ogihara and Tonomura, 1982). The AhhA protein may have similar function, although it remains to be elucidated.

\section{A link between Dd-STATa signaling and the AhhA protein and the cytoskeleton?}

Our finding that the $a h h A$ is down-regulated in the Dd-STATa null mutant at later stages of development (Figs. 1 and 5), suggests that Dd-STATa has a role(s) during culmination of regulating this adducin-like protein. As the protein harbors a putative actinbinding domain, this provides a tool to investigate Dd-STATa function on morphogenesis, through the cytoskeleton.

\section{Materials and Methods}

\section{Cells and growth conditions}

$D$. discoideum $A \times 2$ and Dd-STATanull cells were cultured axenically in $\mathrm{HL} 5$ medium at $22^{\circ} \mathrm{C}$. Cells of Dd-STATanull and SSK395(ahhA)-null strains were grown in HL5 supplemented $10 \mu \mathrm{g} / \mathrm{ml}$ blasticidin S (Kaken Pharmaceutical, Tokyo, Japan). Transformants containing the $\mathrm{Neo}^{\mathrm{R}}$ cassette were selected in HL5 supplemented with $10 \mu \mathrm{g} / \mathrm{ml} \mathrm{G418} \mathrm{(geneticin;}$ ICN Biochemicals Inc., Ohio, USA) and then the concentration of G418 was increased to $20 \sim 80 \mu \mathrm{g} / \mathrm{ml}$.

\section{Genomic DNA cloning}

The genomic sequence of the SSK395(ahhA) gene was obtained via a BLAST search (http://dictybase.org/db/cgi-bin/blast.pl) using the SSK395 cDNA sequence from the Dicty-cDNA database (http:// www.csm.bio.tsukuba.ac.jp/cDNAproject.html) as a search term. The genomic fragment was amplified by PCR using the oligonucleotides 395-G2 (5'-TGC TCT AGA GCA CAT TAG TTT TAA CTC ATT TAG CTC-3') and 395-G6 (5'-CCG CTC GAG CGG CTT GTT TGG TGA AAG AAG CAG CTG-3') as primers (see Fig. 7A) and genomic DNA from the Ax2 strain as the template. A PCR fragment of approximately $1.7 \mathrm{kbp}$, was amplified and subsequently subcloned into PCR4-TOPO (Invitrogen Life Technologies) to yield pTOPO-395.

\section{Construction of targeting vector}

An SSK395(ahhA) targeting vector was constructed by use of the inverse PCR. Both oligonucleotides 395-G1 (5'-CGC GGA TTC GCG ATT AAC TAA TGA AAG GTC ATC ACT ACT TG-3') and 395-G5 (5'CGC GGA TTC GCG AGT TCG TGT CAA ATT GGC AGC AAC-3') contain $B a m H I$ site (underlined) at their 5 ' end, which were used as primers and pTOPO-395 as the template. Amplified fragments were digested with $\mathrm{BamHI}$ and ligated with a $\mathrm{bsr}^{\mathrm{R}}$ cassette digested with BamHI.

\section{Screening of the SSK395(ahhA)-null strains}

The target vector was digested with Not and Spel. After phenol/ chloroform extraction followed by ethanol precipitation of the vector DNA, Ax2 cells were transformed by electroporation. Transformants were selected in 96-wells plates containing HL5 medium supplemented with $10 \mu \mathrm{g} / \mathrm{ml}$ blasticidin S. To screen the clones for those in which the SSK395(ahhA) gene had been successfully targeted, genomic DNA from each clone was amplified by PCR using two pairs of primers 395ORF-2 (5'-CCG CTC GAG TTA ATT TTT AAA AGA ATC ATC TAA TTT TTC AAC AAT TC-3') / Bsr-1 (5'-GCT CCC ACA TCA TGT TTA TTA TCC-3') and 395-G2 / 395-ORF-2 (see Fig. 7A).

\section{Analysis of gene expression by semi-quantitative RT-PCR}

RT-PCR was performed as described previously (Shimada et al., 2004b) by RNA LA PCR Kit (AMV) version 1.1 (TaKaRa, Kyoto, Japan) using a pair of primers 395-ORF-1 (5'-CGG AAT TCA TGA TTT CAA ATA ATA AAT TTA CAA GTA GTG ATG ACC TTT C-3') and 395-ORF2.

\section{Construction of various lacZ fusion construct and expression} construct

The promoter of the SSK395gene was amplified by PCR to add an $X$ bal site at its 5' end and a BgAl site at its 3' end. After digestion with $X b a l$ and $B g A l$, the fragment was gel-purified and subcloned into $X b a l /$ BgAl cut pDd-gal17(H+) (Harwood and Drury, 1990) to make pSSK395::/acZ. For 5 ' deletions, primers corresponding to each deletion point with $X b a l$ site at the 5' end were used to amplify the deleted fragment, then subcloned into pDd-gal17 $(\mathrm{H}+)$ as above.

For construction of human c-myc-tagged SSK395(AhhA) expression vector, pDd-gal $17 \mathrm{H}(+)$ was digested with $B g A l$ and $X$ hol to delete the lac $Z$ gene to produce pDd-gal $17 \mathrm{H}(+) \Delta$ gal. The genomic fragment was amplified by PCR with a pair of oligonucleotides 395-G2 and 395ORF-2 as primers using genomic DNA from Ax2 cells as a template. A DNA fragment, which contains an entire ahhA ORF and its promoter was amplified and subcloned into pCR2.1-TOPO (Invitrogen). After digestion with ECORI, the fragment containing SSK395(ahhA) ORF 
and its promoter was gel purified and ligated into $E c o R I$ site of pDdgal $17 \mathrm{H}(+) \Delta$ gal to yield $\mathrm{pDd}$-ahhA. As a human c-myc epitope EQKLISEEDL, two oligonucleotides corresponding to sense (5'-AGCT GAA CAA AAA CTT ATT TCT GAA GAA GAT CTT GC-3') and antisense (5'-AGCT GC AAG ATC TTC TTC AGA AAT AAG TTT TTG TTC-3') strands were annealed then ligated into an unique Hindlll site of pDd-ahhA to make pDd-ahhA-myc (see Fig. 8). The Hindlll site was located $584 \mathrm{bp}$ downstream from translational start codon of the SSK395(ahhA) ORF. Direction of the inserted double stranded oligonucleotides and the reading frame was confirmed by PCR and sequencing.

\section{Promoter analysis by $\beta$-galactosidase staining}

For the detection of promoter activity, cells transformed with various deleted promoter fragments fused to lacZor ile::/acZ (Detterbeck et al., 1994) reporter plasmids were grown and developed on nitrocellulose filters. Fixation and staining were performed as described (Dingermann et al., 1989).

\section{Western analysis and immunohistochemical staining}

For Western analysis, cells were solubilized in SDS sample buffer containing EDTA-free Complete Mini (Roche, Penzberg, Germany) as proteinase inhibitors. The proteins were analyzed on $5 \sim 20 \%$ gradient SDS-polyacrylamide gels and blotted onto Hybond-ECL filters (Amersham Biosciences, Ltd, UK). The filters were blocked and detected by Promega Proto Blot II AP System with Stabilized Substrate according to the supplied protocol (Promega, WI, USA). Mouse antihuman c-myc monoclonal antibody $9 \mathrm{E} 10$ (1:2,000 dilution, Exalpha Biologicals, Inc., MA, USA) was used as a primary antibody and alkaline phosphatase-conjugated anti-mouse $\lg \mathrm{G}(\mathrm{HL})$ antibody (1:10,000 dilution, Promega) was used as a secondary antibody.

For whole mount immunohistochemical staining, pre-culminant stage multicelllar structures were allowed to settle on poly-L-lysine coated slides and fixed with methanol for 10 min then dried. Samples were incubated with $9 \mathrm{E} 10$ antibody (1:200 dilution, Exalpha Biologicals, Inc.) for $20 \mathrm{hrs}$ in blocking solution. After washing with PBS, they were incubated with Alexa Fluor 568 goat anti-mouse antibody (1:1000 dilution, Invitrogen life technologies, USA) for $20 \mathrm{hrs}$ in PBS. For the detection of F-actin, 170 nM Alexa Fluor 488 phalloidin (Invitrogen life technologies) were used. The samples were mounted and visualized in OLYMPUS fluorescent relief-contrast microscope (model CKX41). The images were taken by Penguiun 150CL Digital Camera System (Pixera, Los Gatos, USA).

\section{Acknowledgements}

This work was supported by a Grant-in-Aid for Scientific Research $C$ from Japanese Society for the Promotion of Science (JSTS) to T. Kawata (\#17570190) and by a Grant-in-Aid for JSPS Fellowships for Young Scientists to N. Shimada (\#17.2751). N.S is a JSTS Research Fellow. We are grateful to Professor Jeffrey G. Williams, University of Dundee, UK, for his critical reading of the manuscript and encouragements on the entire work.

\section{References}

BAUM, B. (2004) Animal development: crowd control. Curr. Biol. 14:R716-R718.

CECCARELLI, A., MAHBUBANI, H. and WILLIAMS, J.G. (1991) Positively and negatively acting signals regulating stalk cell and anterior-like cell differentiation in Dictyostelium. Cel/65:983-989.

COATES, J.C. (2003) Armadillo repeat proteins: beyond the animal kingdom. Trends. Cell Biol. 13:463-471.

COATES, J.C. and HARWOOD, A.J. (2001) Cell-cell adhesion and signal transduction during Dictyostelium development. J. Cell Sci. 114:4349-4358.
CORPET, F. (1988) Multiple sequence alignment with hierarchical clustering Nucl. Acids Res. 16:10881-10890.

DetTERBECK, S., MORANDINI, P., WeTteraUeR, B., BACHMAIR, A., FISCHER, K. and MACWILLIAMS, H.K. (1994) The 'prespore-like cells' of Dictyostelium have ceased to express a prespore gene: analysis using shortlived $\beta$-galactosidases as reporters. Development 120:2847-2855.

DINGERMANN, T., REINDL, N., WERNER, H., HILDEBRANDT, M., NELLEN, W., HARWOOD, A. WILLIAMS, J. and NERKE, K. (1989) Optimization and in situ detection of Escherichia coli $\beta$-galactosidase gene expression in Dictyostelium discoideum. Gene 85:353-362.

EICHINGER, L., PACHEBAT, J.A., GLÖCKNER, G., et al. (2005) The genome of the social amoeba Dictyostelium discoideum. Nature 435:43-57.

GASKELL, M.W., TREFFRY, D., JERMYN, K.A. and WILLIAMS, J.G. (1992) Immuno-localization and separation of multiple prestalk cell types in Dictyostelium. Differentiation 51:171-176.

GRIMSON, M.J., COATES, J.C., REYNOLDS, J.P., SHIPMAN, M., BLANTON R.L. and HARWOOD, A.J. (2000) Adherens junctions and $\beta$-catenin-mediated cell signaling in a non-metazoan organism. Nature 408:727-731.

HARWOOD, A.J. and DRURY, L. (1990) New vectors for expression of the $E$. coli lacZ gene in Dictyostelium. Nucl. Acids Res. 18:4292.

HARWOOD, A.J., EARLY, A. and WILLIAMS, J.G. (1993) A repressor controls the timing and spatial localisation of stalk cell-specific gene expression in Dictyostelium. Development 118:1041-1048.

HUGHES, C.A. and BENNETT, V. (1995) Adducin: a physical model with implications for function in assembly of spectrin-actin complexes. J. Biol. Chem. 270:18990-18996.

HOU, S.X., ZHEN, Z., CHEN, X. and PERRIMON, N. (2002) The JAK/STAT pathway in model organisms: Emerging roles in cell movement. Dev. Cell 3:765-778.

IRANFAR, N., FULLER, D. and LOOMIS, W. F. (2003) Genome-wide expression analyses of gene regulation during early development of Dictyostelium discoideum. Eukaryotic Cel/2:664-670.

JERMYN, K.A., DUFFY, K.T. and WILLIAMS, J.G. (1989) A new anatomy of the prestalk zone of Dictyostelium. Nature 340:144-146

KAWATA,T., SHEVCHENKO, A., FUKUZAWA, M., JERMYN, K.A., TOTTY, N.F. ZHUKOVSKAYA, N.V., STERLING, A.E., MANN, M. and WILLIAMS, J.G. (1997) SH2 signaling in a lower eukaryote: A STAT protein that regulates stalk cell differentiation in Dictyostelium. Cel/89:909-916.

KIMMEL, A.R. and FIRTEL, R.A. (2004) Breaking symmetries: regulation of Dictyostelium development through chemoattractant and morphogen signalresponse. Curr. Opin. Genet. Dev. 14:540-549.

LEPTIN, M. (2005) Gastrulation movements: the logic and the nuts and bolts. Dev. Cel/8:305-320.

LI X., MATSUOKA, Y. and BENNETT, V. (1998) Adducin preferentially recruits spectrin to the fast growing ends of actin filaments in a complex requiring the MARCKS-related domain and a newly defined oligomerization domain. $J$. Biol. Chem. 273:19329-19338.

LIN, B., NASIR, J., MCDONALD, H., GRAHAM, R., ROMMENS, J.M., GOLDBERG Y.P. and HAYDEN, M.R. (1995) Genomic organization of the human alphaadducin gene and its alternately spliced isoforms. Genomics 25:93-99.

MAEDA, M., SAKAMOTO, H., MARUO, T., OGIHARA, S., IRANFAR, N., FULLER, D., MORIO, T., URUSHIHARA, H., TANAKA, Y. and LOOMIS, W.F. (2003) Changing patterns of gene expression in prestalk cell subtypes of Dictyostelium recognised by in situ hybridisation with genes from microarray analyses. Eukaryotic Cel/2:638-645.

MOHANTY, S., JERMYN, K.A., EARLY, A., KAWATA, T., AUBRY, L, CECCARELLI, A., SCHAAP, P., WILLIAMS, J.G. and FIRTEL, R.A. (1999) Evidence that the Dictyostelium Dd-STATa protein is a repressor that regulates commitment to stalk cell differentiation and is also required for efficient chemotaxis. Development 126:3391-3405.

OGIHARA, S. and TONOMURA, Y. (1982) A novel 36,000-dalton actin-binding protein purified from microfilaments in Physarum plasmodis which aggregates actin filaments and blocks actin-myosin interaction. J. Cell Biol. 93:604-614. 
PONTE, E., RIVERO, F., FECHHEIMER, M., NOEGEL, A. and BOZZARO, S. (2000) Severe developmental defects in Dictyostelium null mutants for actinbinding proteins. Mech. Dev. 91:153-161.

RICHARDSON, D.L., LOOMIS, W.F. and KIMMEL, A.L. (1994) Progression of an inductive signal activates sporulation in Dictyostelium discoideum. Development 120: 2891-2900.

ROBINSON, D.N., CANT, K. and COOLEY, L. (1994) Morphogenesis of Drosophila ovarian ring canals. Development 120:2015-2025.

SHIMADA, N., MAEDA, M., URUSHIHARA, H. and KAWATA, T. (2004a) Identification of new modes of Dd-STATa gene expression in Dictyostelium by in situ hybridisation. Int. J. Dev. Biol. 48:679-682.

SHIMADA, N., NISHIO, K., MAEDA, M., URUSHIHARA, H. and KAWATA, T. (2004b) Extracellular matrix family proteins that are potential targets of DdSTATa in Dictyostelium discoideum. J. Plant Res. 117:345-353.
WILLIAMS, H.P. and HARWOOD, A.J. (2003) Cell polarity and Dictyostelium development. Curr. Opin. Microbiol. 6:621-627.

XIA, Y. and KARIN, M. (2004) The control of cell motility and epithelial morphogenesis by Jun kinases. Trends. Cell Biol. 14:94-101.

YUE, L. and SPRADLING, A.C. (1992) hu-li tai shao, a gene required for ring canal formation during Drosophila oogenesis, encodes a homolog of adducin. Genes Dev. 6:2443-2454.

Received: October 2005 Reviewed by Referees: December 2005

Modified by Authors and Accepted for Publication: February 2006

Published Online: May 2006 